\title{
Integrable dispersionless KdV hierarchy with sources
}

\author{
Zhihua Yang*, Ting Xiao and Yunbo Zeng \\ Department of Mathematical Sciences, Tsinghua University, Beijing 100084, P. R. China
}

\begin{abstract}
An integrable dispersionless KdV hierarchy with sources (dKdVHWS) is derived. Lax pair equations and bi-Hamiltonian formulation for dKdVHWS are formulated. Hodograph solution for the dispersionless $\mathrm{KdV}$ equation with sources (dKdVWS) is obtained via hodograph transformation. Furthermore, the dispersionless Gelfand-Dickey hierarchy with sources (dGDHWS) is presented.

PACS number: 02. 03. Ik
\end{abstract}

\section{Introduction}

In recent years, research in the dispersionless hierarchies has become quite active (see, for example, 1]-11] and references therein). Dispersionless hierarchies arise as the quasiclassical limit of the original dispersionfull hierarchies [2]. The operators in the Lax equations for dispersionfull hierarchy are replaced by phase functions for dispersionless hierarchy, commutators are replaced by Poisson brackets and the role of Lax pair equations by dispersionless Lax pair equations. The dispersionless hierarchies have Hamiltonian formulation [1, 3] and many other aspects [4, 5, 6], and several methods of solutions of dispersionless hierarchies have been formulated [7, 8, 9, 10, 11].

The soliton equations with self-consistent sources (SESCS) are another type of integrable models and have important physical applications [12]-28]. There are some ways to derive the SESCS, for example, the Mel'nikov way [12, 18, 19, 21, 30] and the Leon approach [13, 14, 15]. In recent years, SESCS were studied based on the constrained flows of soliton equations which are just the stationary equations of SESCSs [16, 17]. There are several methods for solving the SESCSs, for example, the inverse scattering method [18, 19, 20], the matrix theory [21], the $\bar{\partial}$ method and gauge transformation [22, 23], and

\footnotetext{
*E-mail: yangzhihua@tsinghua.org.cn
} 
the Darboux transformation [17, 24, 25, 26]. By treating the variable $x$ as the evolution parameter and $t$ as the 'spatial' variable respectively, and introducing Jacobi-Ostrogradiski coordinates, the SESCS has a $t$-type Hamiltonian formulation [27, 28].

This paper is devoted to the integrable dispersionless $\mathrm{KdV}$ hierarchy with sources (dKdVHWS). Considering the asymptotic expansion of the wave function, and taking the dispersionless limit of the KdV hierarchy with sources (KdVHWS), we can deduce the dKdVHWS. The Lax pair equations of the dKdVHWS can be deduced by the dispersionless limit of the Lax pair equations of the KdVHWS. Similar to the dKdV hierarchy, the dKdVHWS has bi-Hamiltonian formulation and can be solved via hodograph transformation. The Gelfand-Dickey hierarchy with sources (GDHWS) is the integrable generalization of the Gelfand-Dickey hierarchy, and the corresponding integrable dispersionless hierarchy, i.e. the dGDHWS, can be deduced.

This paper is organized as follows: in Section 2 we review some definitions and results about the KdVHWS. In Section 3 we derive the dKdVHWS as well as its Lax pair equations by taking the dispersionless limit of the KdVHWS. In Section 4, we construct the bi-Hamiltonian formulation of dKdVHWS. Then we derive the hodograph solution for dispersionless KdV equation with sources (dKdVWS) in Section 5. In Section 6, we deduce the integrable dispersionless Gelfand-Dickey hierarchy with sources (dGDHWS). Some conclusion is made in Section 7.

\section{The KdV hierarchy with sources}

We first briefly review some definitions and results about the KdV hierarchy with sources in the framework of Sato theory. Given a pseudo-differential operator (PDO) of the form 29

$$
L=\partial^{2}+u
$$

where $\partial=\frac{\partial}{\partial x}, u=u(x, t), t=\left(t_{3}, t_{5}, \ldots\right)$, and the wave function $\psi=\psi(x, t)$, consider the Lax pair

$$
\begin{gathered}
L \psi=\lambda \psi, \\
\psi_{t_{2 m-1}}=B_{2 m-1} \psi,
\end{gathered}
$$

where $\lambda$ is a parameter, $B_{2 m-1}=\left(L^{\frac{2 m-1}{2}}\right)_{+}, m=1,2,3, \ldots$, and $(A)_{+}$here standing for the differential part of $A$. The compatibility condition of $(2.2 \mathrm{a})$ and $(2.2 \mathrm{~b})$ gives rise to

$$
\frac{\partial L}{\partial t_{2 m-1}}=\left[B_{2 m-1}, L\right],
$$


which is the well-known KdV hierarchy 29]. As was shown in [29], the KdV hierarchy could be written as bi-Hamiltonian systems

$$
u_{t_{2 m-1}}=B_{0} \frac{\delta \mathscr{H}_{2 m+1}}{\delta u}=B_{1} \frac{\delta \mathscr{H}_{2 m-1}}{\delta u}, m=1,2, \ldots,
$$

where $\mathscr{H}_{2 m-1}=\int h_{2 m-1} d x$ is a functional of $u, B_{0}=\partial, B_{1}=\frac{1}{4} \partial^{3}+u \partial+\frac{1}{2} u_{x}$ are Hamiltonian operators, and $\frac{\delta \mathscr{H}_{2 m-1}}{\delta u}$ is the Euler-Lagrange derivative of the Hamiltonian $\mathscr{H}_{2 m-1}$ defined as

$$
\frac{\delta \mathscr{H}_{2 m-1}}{\delta u}=\frac{\delta}{\delta u} \int h_{2 m-1} d x=\sum_{k=0}^{\infty}(-\partial)^{k} \frac{\partial h_{2 m-1}}{\partial u^{(k)}} .
$$

Let us consider [16]

$$
\widetilde{B}_{2 m-1}=B_{2 m-1}+\sum_{k=1}^{n} \psi_{k} \partial^{-1} \phi_{k}, \quad m=1,2,3, \ldots,
$$

where $\psi_{k}=\psi_{k}(x, t)$ and $\phi_{k}=\phi_{k}(x, t)$ satisfying

$$
L \psi_{k}=\lambda_{k} \psi_{k}, L^{*} \phi_{k}=\lambda_{k} \phi_{k}, k=1, \ldots, n
$$

here $L^{*}=(-\partial)^{2}+u=L$ is the adjoint operator of $L$, and $\lambda_{k}$ is a constant, $k=1, \ldots, n$. Then the KdV hierarchy with sources (KdVHWS) [16, 20, 24, 30] can be defined as

$$
\begin{gathered}
\frac{\partial L}{\partial t_{2 m-1}}=\left[\widetilde{B}_{2 m-1}, L\right], \\
L \psi_{k}=\lambda_{k} \psi_{k}, \\
L^{*} \phi_{k}=\lambda_{k} \phi_{k}
\end{gathered}
$$

with the Lax pair given by

$$
\begin{gathered}
L \psi=\lambda \psi, \\
\psi_{t_{2 m-1}}=\widetilde{B}_{2 m-1} \psi,
\end{gathered}
$$

namely, under (2.8b) and (2.8c), the compatibility condition of (2.9a) and (2.9b) gives rise to $(2.8 \mathrm{a})$. 


\section{Dispersionless limit}

Following the procedure introduced in 2, 3, 9, 10, we could derive the dispersionless hierarchy by taking the dispersionless limit of the initial system. Taking $T=\epsilon t, X=\epsilon x$, and thinking of $u(x, t)=u\left(\frac{X}{\epsilon}, \frac{T}{\epsilon}\right)=U(X, T)+O(\epsilon)$ as $\epsilon \rightarrow 0, L$ in (2.1) changes into

$$
L_{\epsilon}=\epsilon^{2} \partial_{X}^{2}+u\left(\frac{X}{\epsilon}, \frac{T}{\epsilon}\right)=\epsilon^{2} \partial_{X}^{2}+U(X, T)+O(\epsilon),
$$

where $\partial_{X}=\frac{\partial}{\partial X}$. It can be proved 3 that

$$
\mathcal{L}=\sigma^{\epsilon}\left(L_{\epsilon}\right)=p^{2}+U
$$

satisfies

$$
\mathcal{L}_{T_{2 m-1}}=\left\{\mathcal{B}_{2 m-1}, \mathcal{L}\right\},
$$

where $\sigma^{\epsilon}$ denotes the principal symbol [2], the bracket $\{$,$\} is the Poison bracket defined$ in $2 D$ 'phase space' $(p, X)$ as

$$
\{A, B\}=\frac{\partial A}{\partial p} \frac{\partial B}{\partial X}-\frac{\partial A}{\partial X} \frac{\partial B}{\partial p},
$$

and $\mathcal{B}_{2 m-1}=\left(\mathcal{L}^{\frac{2 m-1}{2}}\right)_{+}$now refers to nonnegative powers of $p$. We define (3.3) as the dispersionless $\mathrm{KdV}(\mathrm{dKdV})$ hierarchy [3], and the first few equations are expressed as

$$
\begin{gathered}
U_{T_{1}}=U_{X}, \\
U_{T_{3}}=\frac{3}{2} U U_{X}, \\
U_{T_{5}}=\frac{15}{8} U^{2} U_{X}, \\
U_{T_{7}}=\frac{35}{16} U^{3} U_{X}, \ldots
\end{gathered}
$$

As was shown in [3], equation (3.5b) has tri-Hamiltonian formulation as

$$
U_{T_{3}}=\mathcal{D}_{1} \frac{\delta H_{5}}{\delta U}=\mathcal{D}_{2} \frac{\delta H_{3}}{\delta U}=\frac{3}{4} \mathcal{D}_{3} \frac{\delta H_{1}}{\delta U},
$$

where

$$
\mathcal{D}_{1}=2 \partial_{X}, \mathcal{D}_{2}=U \partial_{X}+\partial_{X} U, \mathcal{D}_{3}=U^{2} \partial_{X}+\partial_{X} U^{2},
$$




$$
H_{1}=\int U d X, H_{3}=\frac{1}{4} \int U^{2} d X, H_{5}=\frac{1}{8} \int U^{3} d X .
$$

For the general case, define

$$
H_{2 m-1}=\frac{2}{2 m-1} \operatorname{Tr} \mathcal{L}^{\frac{2 m-1}{2}}
$$

where $\operatorname{Tr} A=\int \operatorname{Res} A d X$, and $\operatorname{Res} A$ is the residue of the general Laurent polynomial of the form $A=\sum_{i=-\infty}^{+\infty} a_{i}(X) p^{i}$, i.e. the coefficient of the $p^{-1}$ term, then the Hamiltonians (3.7) are in involution with respect to any of the three Poisson brackets

$$
\left\{H_{2 m-1}, H_{2 l-1}\right\}_{i}=\int d X \frac{\delta H_{2 m-1}}{\delta U} \mathcal{D}_{i} \frac{\delta H_{2 l-1}}{\delta U}=0, \quad i=1,2,3
$$

and $\mathrm{dKdV}$ hierarchy (3.3) have tri-Hamiltonian formulation

$$
U_{T_{2 m-1}}=\mathcal{D}_{1} \frac{\delta H_{2 m+1}}{\delta U}=\mathcal{D}_{2} \frac{\delta H_{2 m-1}}{\delta U}=\frac{(2 m-1)(2 m-3)}{(2 m-2)^{2}} \mathcal{D}_{3} \frac{\delta H_{2 m-3}}{\delta U}, m=2,3, \ldots
$$

It was also shown in [3] that the solution of $(3.5 \mathrm{~b})$ can be described through the implicit form

$$
U=f\left(X+\frac{3}{2} U T_{3}\right)
$$

where $f$ is an arbitrary function.

In what follows, we derive the dispersionless KdV hierarchy with sources (dKdVHWS).

By taking $T=\epsilon t, X=\epsilon x,(2.8)$ change into

$$
\begin{gathered}
\epsilon L_{\epsilon T_{2 m-1}}=\left[B_{\epsilon(2 m-1)}+\sum_{k=1}^{n} \psi_{k}\left(\frac{X}{\epsilon}, \frac{T}{\epsilon}\right)\left(\epsilon \partial_{X}\right)^{-1} \phi_{k}\left(\frac{X}{\epsilon}, \frac{T}{\epsilon}\right), L_{\epsilon}\right], \\
L_{\epsilon} \psi_{k}\left(\frac{X}{\epsilon}, \frac{T}{\epsilon}\right)=\lambda_{k} \psi_{k}\left(\frac{X}{\epsilon}, \frac{T}{\epsilon}\right), \\
L_{\epsilon}^{*} \phi_{k}\left(\frac{X}{\epsilon}, \frac{T}{\epsilon}\right)=\lambda_{k} \phi_{k}\left(\frac{X}{\epsilon}, \frac{T}{\epsilon}\right),
\end{gathered}
$$

where $B_{\epsilon(2 m-1)}=\left(L_{\epsilon}^{\frac{2 m-1}{2}}\right)_{+}$.

Similar to the dispersionless KP case in [2, 9, 10, we consider the following WKB asymptotic expansion of $\psi_{k}\left(\frac{X}{\epsilon}, \frac{T}{\epsilon}\right)$ and $\phi_{k}\left(\frac{X}{\epsilon}, \frac{T}{\epsilon}\right), k=1,2, \ldots, n$,

$$
\psi_{k}\left(\frac{X}{\epsilon}, \frac{T}{\epsilon}\right) \sim \exp \left\{\frac{S\left(X, T, \lambda=\lambda_{k}\right)}{\epsilon}+\beta_{k 1}+O(\epsilon)\right\}, \epsilon \rightarrow 0,
$$




$$
\phi_{k}\left(\frac{X}{\epsilon}, \frac{T}{\epsilon}\right) \sim \exp \left\{-\frac{S\left(X, T, \lambda=\lambda_{k}\right)}{\epsilon}+\beta_{k 2}+O(\epsilon)\right\}, \epsilon \rightarrow 0,
$$

it can be calculated that

$$
\begin{aligned}
& \psi_{k}\left(\frac{X}{\epsilon}, \frac{T}{\epsilon}\right)\left(\epsilon \partial_{X}\right)^{-1} \phi_{k}\left(\frac{X}{\epsilon}, \frac{T}{\epsilon}\right) \\
= & e^{\beta_{k 1}+\beta_{k 2}}\left[\left(\epsilon \partial_{X}\right)^{-1}+\left(\frac{\partial}{\partial X} S\left(X, T, \lambda=\lambda_{k}\right)+O(\epsilon)\right)\left(\epsilon \partial_{X}\right)^{-2}\right. \\
+ & \left.\left(\left(\frac{\partial}{\partial X} S\left(X, T, \lambda=\lambda_{k}\right)\right)^{2}+O(\epsilon)\right)\left(\epsilon \partial_{X}\right)^{-3}+\ldots\right],
\end{aligned}
$$

as $\epsilon \rightarrow 0$. Therefore, we have

$$
\sigma^{\epsilon}\left(\psi_{k}\left(\frac{X}{\epsilon}, \frac{T}{\epsilon}\right)\left(\epsilon \partial_{X}\right)^{-1} \phi_{k}\left(\frac{X}{\epsilon}, \frac{T}{\epsilon}\right)\right)=\frac{v_{k}}{p-p_{k}},
$$

where

$$
v_{k}=e^{\beta_{k 1}+\beta_{k 2}}, p_{k}=\frac{\partial}{\partial X} S\left(X, T, \lambda=\lambda_{k}\right) .
$$

Taking the principal symbol of both sides of (3.11a), we have

$$
\mathcal{L}_{T_{2 m-1}}=\left\{\mathcal{B}_{2 m-1}+\sum_{k=1}^{n} \frac{v_{k}}{p-p_{k}}, \mathcal{L}\right\}=\left\{\mathcal{B}_{2 m-1}, \mathcal{L}\right\}+\left\{\sum_{k=1}^{n} \frac{v_{k}}{p-p_{k}}, \mathcal{L}\right\},
$$

and the dispersionless limit of (3.11b) and (3.11c) lead to

$$
p_{k}^{2}+U=\lambda_{k}, \frac{\partial}{\partial X}\left(p_{k} v_{k}\right)=0 .
$$

Under (3.16), it can be found that

$$
\left\{\frac{v_{k}}{p-p_{k}}, \mathcal{L}\right\}=-2 v_{k, X},
$$

therefore, the dispersionless limit of (3.11), i.e. dKdVHWS, reads

$$
\begin{gathered}
U_{T_{2 m-1}}=\left\{\mathcal{B}_{2 m-1}, \mathcal{L}\right\}-2 \sum_{k=1}^{n} v_{k, X}, m=1,2,3, \ldots, \\
p_{k}^{2}+U=\lambda_{k}, \\
\frac{\partial}{\partial X}\left(p_{k} v_{k}\right)=0 .
\end{gathered}
$$


Integrating (3.18c) and taking $\lambda_{k}^{m}$ as the integral constants, then we can eliminate $v_{k}$ in (3.18a) and rewrite dKdVHWS in another form

$$
U_{T_{2 m-1}}=\left\{\mathcal{B}_{2 m-1}, \mathcal{L}\right\}-2 \sum_{k=1}^{n}\left(\frac{\lambda_{k}^{m}}{\sqrt{\lambda_{k}-U}}\right)_{X}
$$

If we take the dispersionless limit of (2.9), we will obtain the Lax pair equations of dKdVHWS (3.18) as

$$
\begin{gathered}
p^{2}+U=\lambda \\
p_{T_{2 m-1}}=\left(\mathcal{B}_{2 m-1}+\sum_{k=1}^{n} \frac{v_{k}}{p-p_{k}}\right)_{X}
\end{gathered}
$$

namely, under (3.18b) and (3.18c), the compatibility condition of (3.20a) and (3.20b) gives rise to $(3.18 \mathrm{a})$. We can eliminate $v_{k}$ and $p_{k}$ in (3.20b) and rewrite (3.20) in another form

$$
\begin{gathered}
p^{2}+U=\lambda \\
p_{T_{2 m-1}}=\left(\mathcal{B}_{2 m-1}+\sum_{k=1}^{n} \frac{\lambda_{k}^{m}}{p \sqrt{\lambda_{k}-U}-\left(\lambda_{k}-U\right)}\right)_{X}
\end{gathered}
$$

which are the Lax pair equations of (3.19).

We give two examples in the following, the first one is the dispersionless KdV equation with sources (dKdVWS)

$$
U_{T_{3}}=\frac{3}{2} U U_{X}-2 \sum_{k=1}^{n}\left(\frac{\lambda_{k}^{2}}{\sqrt{\lambda_{k}-U}}\right)_{X}
$$

with Lax pair equations given by

$$
\begin{gathered}
p^{2}+U=\lambda \\
p_{T_{3}}=\left(p^{3}+\frac{3}{2} U p+\sum_{k=1}^{n} \frac{\lambda_{k}^{2}}{p \sqrt{\lambda_{k}-U}-\left(\lambda_{k}-U\right)}\right)_{X} .
\end{gathered}
$$

And the second example is the dispersionless $\mathrm{KdV}(5)$ equation with sources (dKdV(5)WS)

$$
U_{T_{5}}=\frac{15}{8} U^{2} U_{X}-2 \sum_{k=1}^{n}\left(\frac{\lambda_{k}^{3}}{\sqrt{\lambda_{k}-U}}\right)_{X},
$$


with Lax pair equations given by

$$
\begin{gathered}
p^{2}+U=\lambda, \\
p_{T_{5}}=\left(p^{5}+\frac{5}{2} U p^{3}+\frac{15}{8} U^{2} p+\sum_{k=1}^{n} \frac{\lambda_{k}^{3}}{p \sqrt{\lambda_{k}-U}-\left(\lambda_{k}-U\right)}\right)_{X} .
\end{gathered}
$$

\section{Hamiltonian formulation of dKdVHWS}

It is well known that the KdV hierarchy has bi-Hamiltonian formulation [29], the $\mathrm{dKdV}$ hierarchy has tri-Hamiltonian formulation [3], or even further, quasi-Hamiltonian formulation [1, and the KdVHWS has bi-Hamiltonian formulation [27. Motivated by the Hamiltonian formulation of the $\mathrm{dKdV}$ case [3], we would construct the bi-Hamiltonian formulation of the dKdVHWS (3.19).

Let us firstly consider dKdVWS (3.22). Set

$$
A_{k}=2 \lambda_{k}^{2} \int \sqrt{\lambda_{k}-U} d X, B_{k}=2 \lambda_{k} \int \sqrt{\lambda_{k}-U} d X,
$$

then by direct computation we have

$$
\begin{gathered}
\mathcal{D}_{1} \frac{\delta A_{k}}{\delta U}=2 \partial_{X}\left(-\frac{\lambda_{k}^{2}}{\sqrt{\lambda_{k}-U}}\right)=-2\left(\frac{\lambda_{k}^{2}}{\sqrt{\lambda_{k}-U}}\right)_{X} \\
\mathcal{D}_{2} \frac{\delta B_{k}}{\delta U}=\left(U \partial_{X}+\partial_{X} U\right)\left(-\frac{\lambda_{k}}{\sqrt{\lambda_{k}-U}}\right)=-\frac{\lambda_{k}^{2} U_{X}}{\left(\lambda_{k}-U\right)^{3 / 2}}=-2\left(\frac{\lambda_{k}^{2}}{\sqrt{\lambda_{k}-U}}\right)_{X} .
\end{gathered}
$$

Therefore, if we denote

$$
\begin{aligned}
& \widetilde{H}_{3}=H_{3}+\sum_{k=1}^{n} B_{k}=\int d X\left(\frac{1}{4} U^{2}+2 \sum_{k=1}^{n} \lambda_{k} \sqrt{\lambda_{k}-U}\right), \\
& \widetilde{H}_{5}=H_{5}+\sum_{k=1}^{n} A_{k}=\int d X\left(\frac{1}{8} U^{3}+2 \sum_{k=1}^{n} \lambda_{k}^{2} \sqrt{\lambda_{k}-U}\right),
\end{aligned}
$$

then equation (3.22) can be written in two Hamiltonian forms

$$
U_{T_{3}}=\mathcal{D}_{1} \frac{\delta \widetilde{H}_{5}}{\delta U}=\mathcal{D}_{2} \frac{\delta \widetilde{H}_{3}}{\delta U} .
$$

For the dKdVHWS (3.19), denote

$$
\widetilde{H}_{2 m-1}=H_{2 m-1}+2 \sum_{k=1}^{n} \lambda_{k}^{m-1} \int \sqrt{\lambda_{k}-U} d X,
$$


we can directly prove (see Appendix) that the Hamiltonians $\widetilde{H}_{2 m-1}, m=1,2, \ldots$ satisfy

$$
\left\{\widetilde{H}_{2 m-1}, \widetilde{H}_{2 l-1}\right\}_{i}=\int d X \frac{\delta \widetilde{H}_{2 m-1}}{\delta U} \mathcal{D}_{i} \frac{\delta \widetilde{H}_{2 l-1}}{\delta U}=0, \quad i=1,2,
$$

therefore, the dKdVHWS (3.19) have bi-Hamiltonian formulation

$$
U_{T_{2 m-1}}=\mathcal{D}_{1} \frac{\delta \widetilde{H}_{2 m+1}}{\delta U}=\mathcal{D}_{2} \frac{\delta \widetilde{H}_{2 m-1}}{\delta U}, m=1,2, \ldots
$$

\section{$5 \quad$ Hodograph solution for dKdVWS}

In this section, using the hodograph transformation [7, 9, 10, we will derive the hodograph solution for the the dKdVWS (3.22). Following [7] and let $U_{T_{3}}=B(U) U_{X}$, we can find from (3.22) that

$$
B(U)=\frac{3}{2} U-\sum_{k=1}^{n} \frac{\lambda_{k}^{2}}{\left(\lambda_{k}-U\right)^{3 / 2}}
$$

Making the hodograph transformation with the change of variables $\left(X, T_{3}\right) \rightarrow\left(U, T_{3}\right)$ and let $X=X\left(U, T_{3}\right)$, we have

$$
0=\frac{d X}{d T_{3}}=\frac{\partial X}{\partial U} \frac{\partial U}{\partial T_{3}}+\frac{\partial X}{\partial T_{3}}=\frac{\partial X}{\partial U} B U_{X}+\frac{\partial X}{\partial T_{3}},
$$

which implies that

$$
\frac{\partial X}{\partial T_{3}}=-B=-\frac{3}{2} U+\sum_{k=1}^{n} \frac{\lambda_{k}^{2}}{\left(\lambda_{k}-U\right)^{3 / 2}} .
$$

It can be integrated as

$$
X+\left(\frac{3}{2} U-\sum_{k=1}^{n} \frac{\lambda_{k}^{2}}{\left(\lambda_{k}-U\right)^{3 / 2}}\right) T_{3}=F(U)
$$

where $F(U)$ is an arbitrary function of $U$, (5.4) gives an implicit solution of (3.22).

When $F=0$ and $\lambda_{1}=\lambda_{2}=\cdots=\lambda_{n}=0$, dKdVWS (3.22) degenerates to dKdV equation (3.5b), and (5.4) degenerates to the rational solution of (3.5b), $U=-\frac{2 X}{3 T}$.

When $F \neq 0$ and is convertible, then solution of dKdVWS (3.22) can be written through the implicit form

$$
U=F^{-1}\left(X+\left(\frac{3}{2} U-\sum_{k=1}^{n} \frac{\lambda_{k}^{2}}{\left(\lambda_{k}-U\right)^{3 / 2}}\right) T_{3}\right),
$$

which is similar to (3.10). 


\section{The dispersionless Gelfand-Dickey hierarchy with sources}

The well-known Gelfand-Dickey hierarchy with sources (GDHWS) [16] is defined as

$$
\begin{gathered}
\frac{\partial L}{\partial t_{m}}=\left[\widetilde{B}_{m}, L\right]=\left[B_{m}+\sum_{k=1}^{n} \psi_{k} \partial^{-1} \phi_{k}, L\right] \\
L \psi_{k}=\lambda_{k} \psi_{k} \\
L^{*} \phi_{k}=\lambda_{k} \phi_{k}
\end{gathered}
$$

where

$$
L=\partial^{N}+u_{N-2} \partial^{N-2}+\cdots+u_{1} \partial+u_{0}
$$

$u=\left(u_{N-2}, \ldots, u_{0}\right)^{T}, u_{i}=u_{i}(x, t), i=0,1, \ldots, N-2, t=\left(t_{2}, t_{3}, \ldots\right), B_{m}=\left[\left(L^{\frac{1}{N}}\right)^{m}\right]_{+}$, $L^{*}=(-\partial)^{N}+(-\partial)^{N-2} u_{N-2}+\cdots+(-\partial) u_{1}+u_{0}$ is the adjoint operator of $L, \lambda_{k}$ is a constant, $k=1, \ldots, n, \psi_{k}=\psi_{k}(x, t)$ and $\phi_{k}=\phi_{k}(x, t)$, and the Lax pair is given by

$$
\begin{gathered}
L \psi=\lambda \psi, \\
\psi_{t_{m}}=\widetilde{B}_{m} \psi,
\end{gathered}
$$

namely, under (6.1b) and (6.1c), the compatibility condition of (6.3a) and (6.3b) gives rise to $(6.1 \mathrm{a})$.

Following the procedure given above, we can derive the dispersionless Gelfand-Dickey hierarchy with sources (dGDHWS). Taking $T=\epsilon t, X=\epsilon x$, and let $u_{k}\left(\frac{X}{\epsilon}, \frac{T}{\epsilon}\right)=U_{k}(X, T)+$ $O(\epsilon)$ as $\epsilon \rightarrow 0$, then (6.1) change into

$$
\begin{gathered}
\epsilon L_{\epsilon T_{m}}=\left[B_{\epsilon m}+\sum_{k=1}^{n} \psi_{k}\left(\frac{X}{\epsilon}, \frac{T}{\epsilon}\right)\left(\epsilon \partial_{X}\right)^{-1} \phi_{k}\left(\frac{X}{\epsilon}, \frac{T}{\epsilon}\right), L_{\epsilon}\right], \\
L_{\epsilon} \psi_{k}\left(\frac{X}{\epsilon}, \frac{T}{\epsilon}\right)=\lambda_{k} \psi_{k}\left(\frac{X}{\epsilon}, \frac{T}{\epsilon}\right), \\
L_{\epsilon}^{*} \phi_{k}\left(\frac{X}{\epsilon}, \frac{T}{\epsilon}\right)=\lambda_{k} \phi_{k}\left(\frac{X}{\epsilon}, \frac{T}{\epsilon}\right),
\end{gathered}
$$

where

$$
\begin{aligned}
L_{\epsilon}= & \left(\epsilon \partial_{X}\right)^{N}+u_{N-2}\left(\frac{X}{\epsilon}, \frac{T}{\epsilon}\right)\left(\epsilon \partial_{X}\right)^{N-2}+\cdots+u_{1}\left(\frac{X}{\epsilon}, \frac{T}{\epsilon}\right) \epsilon \partial_{X}+u_{0}\left(\frac{X}{\epsilon}, \frac{T}{\epsilon}\right) \\
= & \left(\epsilon \partial_{X}\right)^{N}+\left(U_{N-2}(X, T)+O(\epsilon)\right)\left(\epsilon \partial_{X}\right)^{N-2}+\ldots \\
& \quad+\left(U_{1}(X, T)+O(\epsilon)\right) \epsilon \partial_{X}+U_{0}(X, T)+O(\epsilon),
\end{aligned}
$$


and $B_{\epsilon m}=\left[\left(L_{\epsilon}^{\frac{1}{N}}\right)^{m}\right]_{+}$. Consider the following WKB asymptotic expansion of $\psi_{k}\left(\frac{X}{\epsilon}, \frac{T}{\epsilon}\right)$ and $\phi_{k}\left(\frac{X}{\epsilon}, \frac{T}{\epsilon}\right), k=1, \ldots, n$,

$$
\begin{gathered}
\psi_{k}\left(\frac{X}{\epsilon}, \frac{T}{\epsilon}\right) \sim \exp \left\{\frac{S\left(X, T, \lambda=\lambda_{k}\right)}{\epsilon}+\beta_{k 1}+O(\epsilon)\right\}, \epsilon \rightarrow 0, \\
\phi_{k}\left(\frac{X}{\epsilon}, \frac{T}{\epsilon}\right) \sim \exp \left\{-\frac{S\left(X, T, \lambda=\lambda_{k}\right)}{\epsilon}+\beta_{k 2}+O(\epsilon)\right\}, \epsilon \rightarrow 0,
\end{gathered}
$$

then the principal symbol [2] of (6.4a) arises

$$
\frac{\partial \mathcal{L}}{\partial T_{m}}=\left\{\mathcal{B}_{m}+\sum_{k=1}^{n} \frac{v_{k}}{p-p_{k}}, \mathcal{L}\right\}
$$

where

$$
\mathcal{L}=\sigma^{\epsilon}\left(L_{\epsilon}\right)=p^{N}+U_{N-2} p^{N-2}+\cdots+U_{1} p+U_{0},
$$

$\mathcal{B}_{m}=\sigma^{\epsilon}\left(B_{\epsilon m}\right)$, and $v_{k}=e^{\beta_{k 1}+\beta_{k 2}}, p_{k}=\frac{\partial}{\partial X} S\left(X, T, \lambda=\lambda_{k}\right)$ are obtained by

$$
\sigma^{\epsilon}\left(\psi_{k}\left(\frac{X}{\epsilon}, \frac{T}{\epsilon}\right)\left(\epsilon \partial_{X}\right)^{-1} \phi_{k}\left(\frac{X}{\epsilon}, \frac{T}{\epsilon}\right)\right)=\frac{v_{k}}{p-p_{k}} .
$$

The dispersionless limit of $(6.4 \mathrm{~b})$ and $(6.4 \mathrm{c})$ give rise to

$$
\begin{gathered}
p_{k}^{N}+U_{N-2} p_{k}^{N-2}+\cdots+U_{1} p_{k}+U_{0}=\lambda_{k}, \\
\frac{\partial}{\partial X}\left(\left.v_{k} \cdot \frac{\partial \mathcal{L}}{\partial p}\right|_{p=p_{k}}\right)=0,
\end{gathered}
$$

(6.7) together with (6.10a) and (6.10b) give rise to the dispersionless Gelfand-Dickey hierarchy with sources (dGDHWS)

$$
\begin{gathered}
\frac{\partial \mathcal{L}}{\partial T_{m}}=\left\{\mathcal{B}_{m}+\sum_{k=1}^{n} \frac{v_{k}}{p-p_{k}}, \mathcal{L}\right\}, \\
p_{k}^{N}+U_{N-2} p_{k}^{N-2}+\cdots+U_{1} p_{k}+U_{0}=\lambda_{k}, \\
\frac{\partial}{\partial X}\left(\left.v_{k} \cdot \frac{\partial \mathcal{L}}{\partial p}\right|_{p=p_{k}}\right)=0,
\end{gathered}
$$

whose Lax pair equations are given by

$$
p^{N}+U_{N-2} p^{N-2}+\cdots+U_{1} p+U_{0}=\lambda
$$




$$
p_{T_{m}}=\left(\mathcal{B}_{m}+\sum_{k=1}^{n} \frac{v_{k}}{p-p_{k}}\right)_{X}
$$

namely, under (6.11b) and (6.11c), the compatibility condition of (6.12a) and (6.12b) gives rise to $(6.11 \mathrm{a})$.

Under $(6.11 b)$ and $(6.11 c)$, it can be found by a tedious computation that

$$
\left\{\frac{v_{k}}{p-p_{k}}, \mathcal{L}\right\}=a_{N-2} p^{N-2}+a_{N-3} p^{N-3}+\cdots+a_{0}
$$

where $a_{N-2}=-N v_{k, X}, a_{N-3}=-N\left(v_{k} p_{k}\right)_{X}$, and for $i=4, \ldots, N$,

$$
a_{N-i}=-v_{k} \sum_{j=1}^{i-3} j p_{k}^{j-1} U_{N+1-i+j, X}-\sum_{l=2}^{i-2}(N-l)\left(v_{k} p_{k}^{i-2-l}\right)_{X} U_{N-l}-N\left(p_{k}^{i-2} v_{k}\right)_{X} .
$$

When $N=2$, we have $\mathcal{L}=p^{2}+U$, and (6.13) is the same as (3.17).

Similar to the dKdVHWS, the dGDHWS possess bi-Hamiltonian formation and their solutions can be obtained via hodograph transformation.

\section{Conclusion}

We derive dKdVHWS by taking the dispersionless limit of KdVHWS, meanwhile, Lax pair equations of the dKdVHWS can be obtained by taking the dispersionless limit of the corresponding dispersionfull equations. We have constructed the bi-Hamiltonian formulation of the dKdVHWS, and have obtained the implicit solutions of the dKdVWS via the hodograph transformation. For the generalization case, we have deduced the dGDHWS which also possess bi-Hamiltonian formulation and can be solved via the hodograph transformation.

\section{Acknowledgment}

This work was supported by the Chinese Basic Research Project "Nonlinear Science". 


\section{Appendix}

Here we give the proof of involution relation of the Hamiltonians $\widetilde{H}_{2 m-1}(4.4)$.

Since

$$
\begin{aligned}
\left\{\widetilde{H}_{2 m-1}, \widetilde{H}_{2 l-1}\right\}_{1} & =\left\{\widetilde{H}_{2 m-1}, \widetilde{H}_{2 l-3}\right\}_{2}=-\left\{\widetilde{H}_{2 l-3}, \widetilde{H}_{2 m-1}\right\}_{2}=-\left\{\widetilde{H}_{2 l-3}, \widetilde{H}_{2 m+1}\right\}_{1} \\
& =\left\{\widetilde{H}_{2 m+1}, \widetilde{H}_{2 l-3}\right\}_{1}=\cdots=\left\{\widetilde{H}_{2 m+2 l-3}, \widetilde{H}_{1}\right\}_{1},
\end{aligned}
$$

it suffices to prove that for any $m \geq 1,\left\{\widetilde{H}_{2 m-1}, \widetilde{H}_{1}\right\}_{1}=0$. We can directly calculate that

$$
\begin{aligned}
& \left\{\widetilde{H}_{2 m-1}, \widetilde{H}_{1}\right\}_{1} \\
= & \int \frac{\delta \widetilde{H}_{2 m-1}}{\delta U} \mathcal{D}_{1} \frac{\delta \widetilde{H}_{1}}{\delta U} d X \\
= & \int\left(\frac{\delta H_{2 m-1}}{\delta U}-\sum_{k=1}^{n} \lambda_{k}^{m-1} \frac{1}{\sqrt{\lambda_{k}-U}}\right) 2 \partial_{X}\left(1-\sum_{k=1}^{n} \frac{1}{\sqrt{\lambda_{l}-U}}\right) d X \\
= & -\int\left(\frac{\delta H_{2 m-1}}{\delta U}-\sum_{k=1}^{n} \lambda_{k}^{m-1} \frac{1}{\sqrt{\lambda_{k}-U}}\right) \sum_{l=1}^{n} \frac{U_{X}}{\sqrt{\lambda_{l}-U}\left(\lambda_{l}-U\right)} d X .
\end{aligned}
$$

Since all $\frac{\delta H_{2 m-1}}{\delta U}, m=1,2, \ldots$ are of the form $c U^{s}$, where $c$ are constants, and $s \in \mathcal{N}$, it suffices to prove

$$
\begin{gathered}
\int \frac{U^{s} U_{X}}{\sqrt{\lambda_{l}-U\left(\lambda_{l}-U\right)}} d X=0, \quad l=1, \ldots, n \\
\int \frac{U_{X}}{\sqrt{\lambda_{k}-U} \sqrt{\lambda_{l}-U}\left(\lambda_{l}-U\right)} d X=0, \quad k, l=1, \ldots, n .
\end{gathered}
$$

For $(I)$, let $F_{l}=\sqrt{\lambda_{l}-U}$, then $U=\lambda_{l}-F_{l}^{2}, U_{X}=-2 F_{l} F_{l, X}$ and

$$
\int \frac{U^{s} U_{X}}{\sqrt{\lambda_{l}-U}\left(\lambda_{l}-U\right)} d X=-2 \int \frac{\left(\lambda_{l}-F_{l}^{2}\right)^{s}}{F_{l}^{2}} d F_{l}
$$

which are all zero for the reason that $\frac{\left(\lambda_{l}-F_{l}^{2}\right)^{s}}{F_{l}^{2}}$ are rational polynomials of $F_{l}^{2}$ and so $\frac{\left(\lambda_{l}-F_{l}^{2}\right)^{s}}{F_{l}^{2}} d F_{l}$ are total differentials.

For $(I I)$, when $k=l$, then $(I I)$ obviously holds; when $k \neq l$, let $G_{l}=\lambda_{l}-U$, then 
$U_{X}=-G_{l, X}$, and

$$
\begin{aligned}
& \int \frac{U_{X}}{\sqrt{\lambda_{k}-U \sqrt{\lambda_{l}-U}\left(\lambda_{l}-U\right)}} d X \\
= & \int \frac{-G_{l, X}}{\sqrt{\lambda_{k}-\lambda_{l}+G_{l}} \sqrt{G_{l}} G_{l}} d X \\
= & \int \frac{-G_{l, X}}{G_{l}^{2} \sqrt{\frac{\lambda_{k}-\lambda_{l}}{G_{l}}+1}} d X \\
= & \frac{1}{\lambda_{k}-\lambda_{l}} \int \frac{1}{\sqrt{\frac{\lambda_{k}-\lambda_{l}}{G_{l}}+1}} d\left(\frac{\lambda_{k}-\lambda_{l}}{G_{l}}\right),
\end{aligned}
$$

set $\frac{\lambda_{k}-\lambda_{l}}{G_{l}}=\tan ^{2} H_{k l}$, then $1+\frac{\lambda_{k}-\lambda_{l}}{G_{l}}=\sec ^{2} H_{k l}, d\left(\frac{\lambda_{k}-\lambda_{l}}{G_{l}}\right)=2 \tan _{k l} \sec ^{2} H_{k l} d H_{k l}$, and

$$
\frac{1}{\sqrt{\frac{\lambda_{k}-\lambda_{l}}{G_{l}}+1}} d\left(\frac{\lambda_{k}-\lambda_{l}}{G_{l}}\right)=\frac{2 \tan H_{k l} \sec ^{2} H_{k l} d H_{k l}}{\sec H_{k l}}=2 d\left(\sec H_{k l}\right),
$$

which are total differentials, and this proves $(I I)$.

\section{References}

[1] Brunelli J C 2000 Braz. J. Phys. 30455

[2] Takasaki T and Takebe T 1995 Rev. Math. Phys. 7743

[3] Brunelli J C 1996 Rev. Math. Phys. 81041

[4] Chen Y T and Tu M H 2003 Lett. Math. Phys. 63125

[5] Zheltukhin K 2002 Phys. Lett. A 297402

[6] Alagesan T Chung Y and Nakkeeran K 2004 Chaos, Solitons and Fractals 2163

[7] Kodama Y 1988 Phys. Lett. A 129223

[8] Kodama Y 1989 Phys. Lett. A 135171 
[9] Xiao T and Zeng Y B 2006 Phys. Lett. A 349128

[10] Xiao T and Zeng Y B 2006 J. Nlin. Math. Phys. at press

[11] Chen Y T and Tu M H 2003 J. Phys. A: Math. Gen. 369875

[12] Mel'nikov V K 1990 J. Math. Phys. 311106

[13] Leon J1988 J. Math. Phys. 292012

[14] Leon J and Latifi A 1990 J. Phys. A 231385

[15] Leon J 1990 Phys. Lett. A 144444

[16] Antonowicz M 1992 Phys. Lett. A 16547

[17] Zeng Y B 1994 Phys. D 73171

[18] Mel'nikov V K 1988 Phys. Lett. A 133493

[19] Mel'niklov V K 1992 Phys. Lett. A 8133

[20] Lin R L, Zeng Y B and Ma W X 2001 Phys. A 291287

[21] Mel'nikov V K 1989 Comm. Math. Phys. 120451

[22] Doktorov E V and Shchesnovich V S 1995 Phys. Lett. A 207153

[23] Shchesnovich V S and Doktorov E V 1996 Phys. Lett. A 21323

[24] Zeng Y B, Ma W X and Shao Y S 2001 J. Math. Phys. 422113

[25] Zeng Y B, Shao Y J and Xue W M 2003 J. Phys. A: Math. Gen. 365035

[26] Zeng Y B, Shao Y J 2005 J. Phys. A: Math. Gen. 382441

[27] Blaszak M 1995 J. Math. Phys. 364826

[28] Zeng Y B 1999 Phys. A 262405

[29] Dickey L A 2002 Soliton equations and Hamiltonian systems (2nd ed.)(World Scientific: Singapore)

[30] Mel'nikov V K 1990 Nonlinear evolution equations and dynamical systems (Berlin: Springer) 